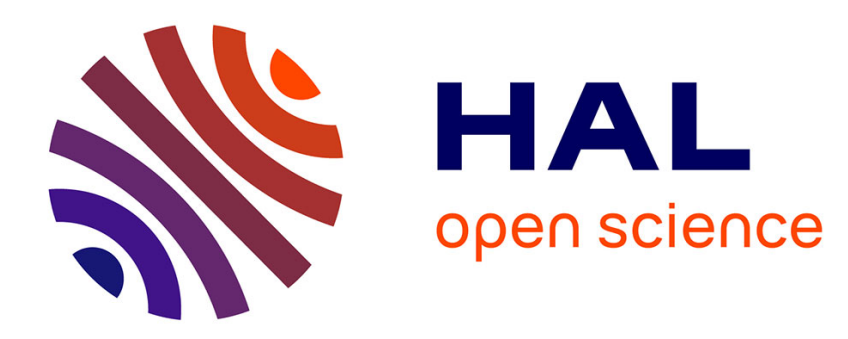

\title{
STANDARDIZATION OF BINAURAL MEASUREMENT TECHNIQUE
}

\author{
K. Genuit
}

\section{To cite this version:}

K. Genuit. STANDARDIZATION OF BINAURAL MEASUREMENT TECHNIQUE. Journal de Physique IV Proceedings, 1992, 02 (C1), pp.C1-405-C1-407. 10.1051/jp4:1992187 • jpa-00251259

\section{HAL Id: jpa-00251259 https://hal.science/jpa-00251259}

Submitted on 1 Jan 1992

HAL is a multi-disciplinary open access archive for the deposit and dissemination of scientific research documents, whether they are published or not. The documents may come from teaching and research institutions in France or abroad, or from public or private research centers.
L'archive ouverte pluridisciplinaire HAL, est destinée au dépôt et à la diffusion de documents scientifiques de niveau recherche, publiés ou non, émanant des établissements d'enseignement et de recherche français ou étrangers, des laboratoires publics ou privés. 


\title{
STANDARDIZATION OF BINAURAL MEASUREMENT TECHNIQUE
}

\author{
K. GENUIT \\ HEAD Acoustics GmbH, Kaiserstr. 100, D-5120 Herzogenrath 3, Germany
}

\section{Standardization of Binaural Measurement Technique}

For many years various Artificial Heads have been available for different applications:

\author{
Music recordings \\ Measurements of hearing aids \\ Subjective sound diagnosis thru A/B-comparison
}

The Artificial Heads were designed for different areas of application. An Artificial Head designed for music recordings consisted of a head simulation with two built-in studio microphones in the left and right ear simulator. An Artificial Head designed for measuring the transfer functions of hearing aids consists of a dummy including torso, shoulder simulation and a built-in ear simulator for simulating the human ear canal input impedance and for recording the sound pressure in a reference plane comparable to the human eardrum. Usually, such an Artificial Head was a one-channel system, i.e. it was not suited for binaural technology. The Artifical Head for subjective sound judgement is always a two-channel system. As playback is made via headphone and the individual ear canal entrance with eardrum is included in the chain of transmission, this system must not include an ear simulator during the recording. Hence it follows that different systems have been available according to the applications in question. Up to now standardizations have been set up only for measuring the transfer functions of hearing aids. In addition to the ANSI 3.36 standard there is only the so-called IEC Report 959 which constitutes the basis of various considerations regarding standardization in the field of CCITT and DIN.

At the time the first Artificial Head was developed nobody had given any thought to the question of whether the transfer characteristics of an Aritificial Head microphone had to be corrected, adapted or changed in any way by means of electrical filters. It was taken for granted that the microphone should be positioned according to the human eardrum. Depending on the application of the Artificial Head technology, such a position is not suitable. If the Artificial Head Measurement System is used to measure sound signals as they are perceived by human hearing, e.g., to determine the damaging effect of sounds on the hearing or to determine the transfer functions of hearing aids, telephone systems, headphones and so forth, such a position of the microphone is appropriate, as then the acoustic input impedance is simulated by the ear canal entrance. If, however, the recorded Artificial Head signals are to be reproduced via headphone or loudspeaker for human-hearing adequate judgements, a mistake creeps in, because the signals pass the ear canal twice, once during the recording and once during the playback. Furthermore, the microphone signals are not compatible with conventional measurement microphones, as the linear distortions, caused by the ear canal resonances, are leading to a strongly frequency-dependent level.

When in 1980 the engineers of a car manufacturer recognized that sound quality could not be determined or compared in terms of measurement technique using conventional measurement procedures, they had the idea to use Artificial Head stereophony instead of standard measurement microphones. During the first tests 
with the Artificial Head available at that time, direct listening comparisons between the original sound and the Artificial Head signal reproduced via headphone revealed that a considerable difference could be perceived. In cooperation with the Institute for Electrical Communications Engineering of the Aachen University a new Artificial Head Measurement System for subjective sound judgement was developed which should guarantee the faithful playback of ear signals via headphone. Meanwhile it was common knowledge that the directional pattern of an Artificial Head did not depend on the impedance of the ear canal entrance. Therefore, the simulation of the ear canal and the impedance of the eardrum was not taken into account. Moreover, it was generally known that the directional pattern of an Artificial Head was mainly determined by the position of geometric parts, such as torso, shoulder, head, outer ear and ear canal entrance. This was the reason why the head simulation was made according to the head of a person who had an average directional pattern, ascertained during a lot of measurements and listening tests.

For reasons of compatibility with conventional measurement techniques this Artificial Head was free-field equalized, i.e. for frontal sound incidence in an anechoic environment a filter was designed, the transfer function of which was for this sound situation inverse to the transfer function of the Artificial Head, so that the network of the Artificial Head and this filter showed a frequency-independent transfer function. This Artificial Head was exclusively used for subjective noise diagnosis and analysis, i.e. for archiving and documentation of sounds to conduct A/B-hearing tests, but also for subjective recognition of annoying sound components by spectral manipulation of the recorded Artificial Head microphone signals. Meanwhile the Artificial Head technology has become more and more important and is now used not only for subjective but also for objective sound measurement technique, e.g., for measuring the noise annoyance, telephone systems, to determine the attenuation characteristics of hearing protectors and so forth. Efforts are being made to use the Artificial Head technology also for a human-hearing adequate noise evaluation of working places. These attempts required a standardization of the Artificial Head technology. In the meantime it was known in how far the outer geometry of an Artificial Head microphone could be simplified without loosing an average directional pattern. Thus, it was possible to develop an Artificial Head that corresponds to a mathematically describable geometry with an average directional pattern representing that of human hearing. The outer dimensions comply with international standards, the free-field outer-ear transfer function and the directional pattern are in accordance with the IEC 959 Report.

If this Artificial Head Measurement System is not only used for measuring the attenuation characteristics of hearing protectors or for the transfer functions of hearing aids, but also for the subjective and objective sound measurement technique, it is advisable for reasons of compatibility with standard measurement techniques, to assign an equalization to this Artificial Head Measurement System as well. As free-field conditions are better reproducible, the reference to the free-field was chosen. For some time thoughts have also been given to the question of whether the free-field equalization is suited for the Artificial Head microphone. There are numerous sound situations which do not agree with this free-field reference measurement, i.e. anechoic environment, sound source exactly from the front. A lot of suggestions have been made to choose an alternative equalization, e.g., an average equalization in the entire sound incidence range $\pm 30^{\circ}$ from the front or the so-called diffuse-field equalization, i.e. the Artificial Head is in a diffuse sound field where the sound is coming evenly from all directions. Some years ago HEAD acoustics developed a different kind of equalization. The transfer function of an Artificial Head can be divided into a directiondependent and a direction-independent part. The direction-dependent part is caused by diffractions and reflections at the torso, shoulder, head and outer ear. The direction-independent components are caused by resonances at the cavum conchae and ear canal entrance. HEAD acoustics developed the so-called IDequalization (independent of direction) which compensates only the direction-independent part of the Artificial Head transfer function. In particular for the Artificial Head from HEAD acoustics (HMS II) the direction-independent components can be determined precisely, as the head is based on a mathematically describable, simplified geometric simulation.

For the human-hearing equivalent judgement of sound events it is not important whether the recording has been made with free-field or ID-equalization, as the headphones have the corresponding free-field or IDequalization, measured at the HMS II, so that every time there are the same ear signals at the ear of the listening person. When analysing in terms of measurement technique, certain differences, particularly in the frequency range around $8 \mathrm{kHz}$, may occur. Practice shows that in numerous sound situations the IDequalization is better compatible with conventional measurement microphones than the free-field equalization, especially when examining sounds having a power density spectrum exceeding $8 \mathrm{kHz}$. When analysing noise inside cars, however, the difference between free-field and ID-equalization can be ignored, because the signal components can mainly be found in the low-frequency spectral range. 
In the following two different cases of sound exposure will show why the standardization of binaural measurement technique requires a suitable equalization by Artificial Head microphone signals. The introduction of a new measurement technology is much easier when it is a logical extension of existing conventional methods. Figs. 1 and 2 show comparative measurements in a free-field (Fig. 1) and a diffusefield sound situation (Fig. 2). The magnitude spectra and the A-weighted SPL are compared. Firstly, they were measured with a normal measurement microphone (curve a) and secondly with two different Artificial Head Measurement Systems complying with IEC Report 959. The system corresponding to curve b was additionally equipped with free-field equalization (Fig. 1) and ID-equalization (Fig. 2). The two Figs. reveal that a suitable equalization is needed to allow an adequate compatibility of Artificial Head measurement technology with conventional methods.

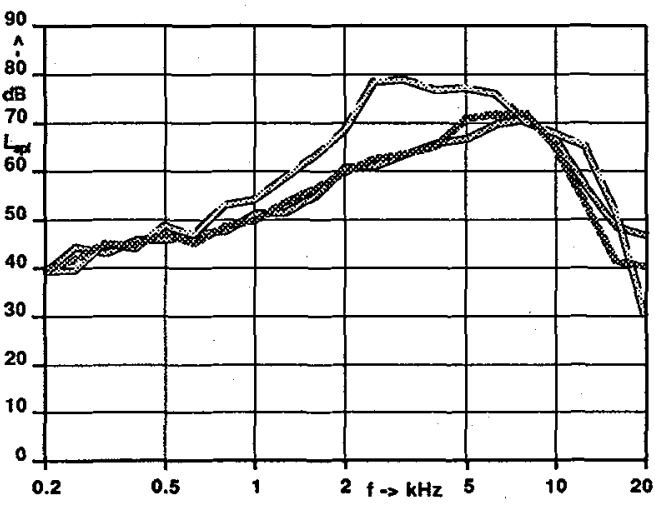

Fig. 1: Comparison of the magnitude spectra in a free-field sound situation

a) Standard microphone, $\mathrm{L}_{\mathrm{SPL}}=80 \mathrm{~dB}(\mathrm{~A})$

b) Artificial Head according to IEC 959 but with free-field equalization, $\mathrm{L}_{\mathrm{SPL}}=80 \mathrm{~dB}(\mathrm{~A})$

c) Artificial Head only according to IEC 959 without any equalization, $L_{S P L}=90 \mathrm{~dB}(\mathrm{~A})$

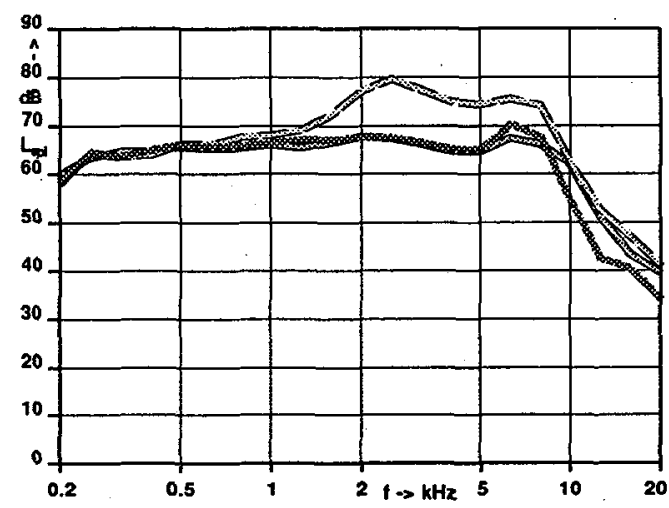

Fig. 2: Comparison of the magnitude spectra in a diffuse-field sound situation

a) Standard microphone, $\mathrm{L}_{\mathrm{SPL}}=79,2 \mathrm{~dB}(\mathrm{~A})$

b) Artificial Head according to IEC 959 but with independent-of-direction equalization, $\mathrm{L}_{\mathrm{SPL}}=$ 79,6 dB(A)

c) Artificial Head only according to IEC 959 without any equalization, $\mathrm{L}_{S P L}=88,6 \mathrm{~dB}(\mathrm{~A})$ 